\title{
High-speed dynamic-mode atomic force microscopy imaging of polymers: an adaptive multiloop-mode approach
}

\author{
Juan Ren ${ }^{1}$ and Qingze Zou ${ }^{* 2}$
}

\author{
Full Research Paper \\ Address: \\ ${ }^{1}$ Department of Mechanical Engineering, lowa State University, 2030 \\ Black Engineering, Ames, IA 50011, USA and 2Department of \\ Mechanical and Aerospace Engineering, Rutgers University, 98 Brett \\ Rd, Piscataway, NJ 08854, USA

\section{Email:} \\ Juan Ren - juanren@iastate.edu; Qingze Zou* - \\ qzzou@rci.rutgers.edu \\ * Corresponding author \\ Keywords: \\ adaptive multiloop mode; atomic force microscopy (AFM); \\ heterogeneous polymer sample; tapping-mode imaging
}

Beilstein J. Nanotechnol. 2017, 8, 1563-1570.

doi:10.3762/bjnano.8.158

Received: 16 February 2017

Accepted: 03 July 2017

Published: 02 August 2017

This article is part of the Thematic Series "Advanced atomic force microscopy".

Guest Editor: T. Glatzel

(c) 2017 Ren and Zou; licensee Beilstein-Institut.

License and terms: see end of document.

\begin{abstract}
Adaptive multiloop-mode (AMLM) imaging to substantially increase (over an order of magnitude) the speed of tapping-mode (TM) imaging is tested and evaluated through imaging three largely different heterogeneous polymer samples in experiments. It has been demonstrated that AMLM imaging, through the combination of a suite of advanced control techniques, is promising to achieve high-speed dynamic-mode atomic force microscopy imaging. The performance, usability, and robustness of the AMLM in various imaging applications, however, is yet to be assessed. In this work, three benchmark polymer samples, including a PS-LDPE sample, an SBS sample, and a Celgard sample, differing in feature size and stiffness of two orders of magnitude, are imaged using the AMLM technique at high-speeds of $25 \mathrm{~Hz}$ and $20 \mathrm{~Hz}$, respectively. The comparison of the images obtained to those obtained by using TM imaging at scan rates of $1 \mathrm{~Hz}$ and $2 \mathrm{~Hz}$ showed that the quality of the $25 \mathrm{~Hz}$ and $20 \mathrm{~Hz}$ AMLM imaging is at the same level of that of the $1 \mathrm{~Hz}$ TM imaging, while the tip-sample interaction force is substantially smaller than that of the $2 \mathrm{~Hz}$ TM imaging.
\end{abstract}

\section{Introduction}

In this paper, the adaptive-multiloop imaging mode of atomic force microscopy (AFM) is tested and evaluated by imaging three largely different heterogeneous polymer samples. AMLM imaging substantially increases the speed of tapping mode (TM) imaging (by over an order of magnitude) while preserving the advantages of TM imaging over contact mode (CM) imaging [1]. Although TM imaging is the de facto most widely used imaging technique of AFM [2,3], the slow speed (throughput) of TM imaging has become its major limit and bottleneck [4,5]. It is challenging to achieve high-speed TM imaging because an increase of the speed can cause a loss of the tip-sample interaction and/or the annihilation of the cantilever tapping vibration, particularly when the imaging size is large. Existing efforts on high-speed TM imaging [6-9] only led to a speed increase up to 
three times at the cost of a substantially (over five times) increased imaging force. By using the AMLM imaging mode, it is aimed to achieve high-speed dynamic-mode AFM imaging while maintaining the tip-sample interaction force similar as that in low-speed TM imaging.

The speed increase of TM imaging is limited by the control mechanism applied $[4,6]$. Due to the time delay inevitably induced into the feedback loop for maintaining the RMS tapping amplitude during imaging, errors in tracking the sample topography can quickly result in loss of the tip-sample contact and annihilation of the probe tapping when the imaging speed increases $[3,6]$. This is because the tapping amplitude is sensitive and highly nonlinear with respect to the tip-sample distance $[10,11]$. The speed of TM-imaging might be increased through either hardware [12-14] or software (algorithms) improvement $[6,8,15,16]$. However, the existing hardware improvements via the use of high-bandwidth piezo actuators and cantilever are only applicable for small-size imaging (less than $30 \%$ of the imaging size of regular AFMs [14]), and the existing algorithm improvement based on advanced control techniques may lead to a potential sample deformation and damage due to the lack of control of the tip-sample interaction force $[7,8,16]$. Therefore, current efforts to high-speed dynamic-mode AFM imaging only led to rather limited success.

The presented AMLM imaging approach can achieve highspeed TM imaging for both large- and small-size imaging while maintaining the image quality and keeping the average tip-sample interaction force at the minimum level for stable cantilever tapping. AMLM imaging is fundamentally different from current efforts to high-speed TM imaging $[6,8,15,16]$ insofar through the introduction of the control of the averaged cantilever deflection (the TM deflection) - in addition to the transitional RMS amplitude feedback control, along with an online iterative feedforward control to track the sample topography. Although this AMLM technique has been proposed recently [1], imaging results of only one polymer sample at large scanning size $(50 \mu \mathrm{m})$ were obtained and presented. The performance, usability, and particularly, robustness of the AMLM technique for a variety of materials of different topography characteristics and different heterogeneous material properties, and at different imaging sizes have not been yet elucidated. As a result, the AMLM imaging as a highly efficient dynamic-mode AFM imaging technique for wide implementations in practice is still yet to be established. Therefore, it is necessary and crucial to test and evaluate the AMLM technique in various imaging scenarios.

In this work, we present the implementation and evaluation of the AMLM technique by imaging three types of polymer samples different in both topography features and heterogeneous mechanical properties. The three samples, made of polystyrene-low-density polyethylene (PS-LDPE), styrene-butadiene-styrene (SBS) and celgard, respectively, have over two orders of magnitude different lateral feature sizes, ranging from $10 \mathrm{~nm}$ (Celgard) to $100 \mathrm{~nm}$ (SBS) and up to $2 \mu \mathrm{m}$ (PS-LDPE), respectively. The elasticity also differs over two orders of magnitude, ranging from $20 \mathrm{MPa}$ (SBS) to $2 \mathrm{GPa}$ (PS). These samples, with largely heterogeneous mechanical properties varying by two orders of magnitude on the same sample, have been widely used as benchmark samples for testing and evaluating AFM imaging. Results of high-speed AMLM imaging $(20 \mathrm{~Hz}$ and $25 \mathrm{~Hz}$ ) on these three samples are evaluated and compared to those of TM imaging at much lower speeds $(2.5 \mathrm{~Hz}$ and $1 \mathrm{~Hz})$ for three largely different imaging sizes $(50 \mu \mathrm{m}, 5 \mu \mathrm{m}$, and $4 \mu \mathrm{m}$ ). All of the imaging results showed that the AMLM imaging can maintain the same image quality at a scan rate of $25 \mathrm{~Hz}$ (with the corresponding probe velocity at $2.5 \mathrm{~mm} / \mathrm{sec}$.) as TM imaging at $1 \mathrm{~Hz}$. The tapping amplitude error and the tip-sample interaction force were reduced by over $15 \%$ and $27 \%$, respectively, from those of TM imaging at scan rates 10 -fold slower $(2.5 \mathrm{~Hz}$ and $2 \mathrm{~Hz}$ ). Therefore, through this work, the AMLM clearly stood the test as an efficient high-speed dynamic-mode AFM-imaging technology for practical applications.

\section{Results and Discussion}

AMLM imaging results of the three samples at the scan rates of $25 \mathrm{~Hz}$ (for the PS-LDPE and the SBS samples) and $20 \mathrm{~Hz}$ (for the Celgard sample) are compared to those obtained using TM imaging at the scan rate of $2.5 \mathrm{~Hz}$ (for the PS-LDPE and the SBS samples) and $2 \mathrm{~Hz}$ (for the Celgard sample) in Figure 1, Figure 2, and Figure 3, respectively, where the scanning size was $50 \mu \mathrm{m}$ (PS-LDPE), $5 \mu \mathrm{m}$ (SBS), and $4 \mu \mathrm{m}$ (celgard), respectively. The sample topography, tapping-amplitude-ratio error, mean tip-sample interaction force, and phase images are also compared. Moreover, zoomed-in views of the topography images of the Celgard sample are compared in Figure 4, and the cross-section comparison of the sample topography and the mean tip-sample interaction force are compared for the PS-LDPE and the celgard samples in Figure 5 and Figure 6, respectively. The sample topography obtained by $1 \mathrm{~Hz}$ TM imaging was used as reference, and the mean tip-sample interaction force was computed using Equation 5.

The imaging results demonstrate the efficacy of AMLM imaging for high-speed dynamic-mode imaging. As shown in Figure 1-Figure 3, the quality of all the AMLM images was clearly improved over that of the TM images at $2.5 \mathrm{~Hz}$ and $2 \mathrm{~Hz}$, and was about the same as that of $1 \mathrm{~Hz}$ TM imaging. Compared to the $1 \mathrm{~Hz}$ TM imaging results as the reference the 

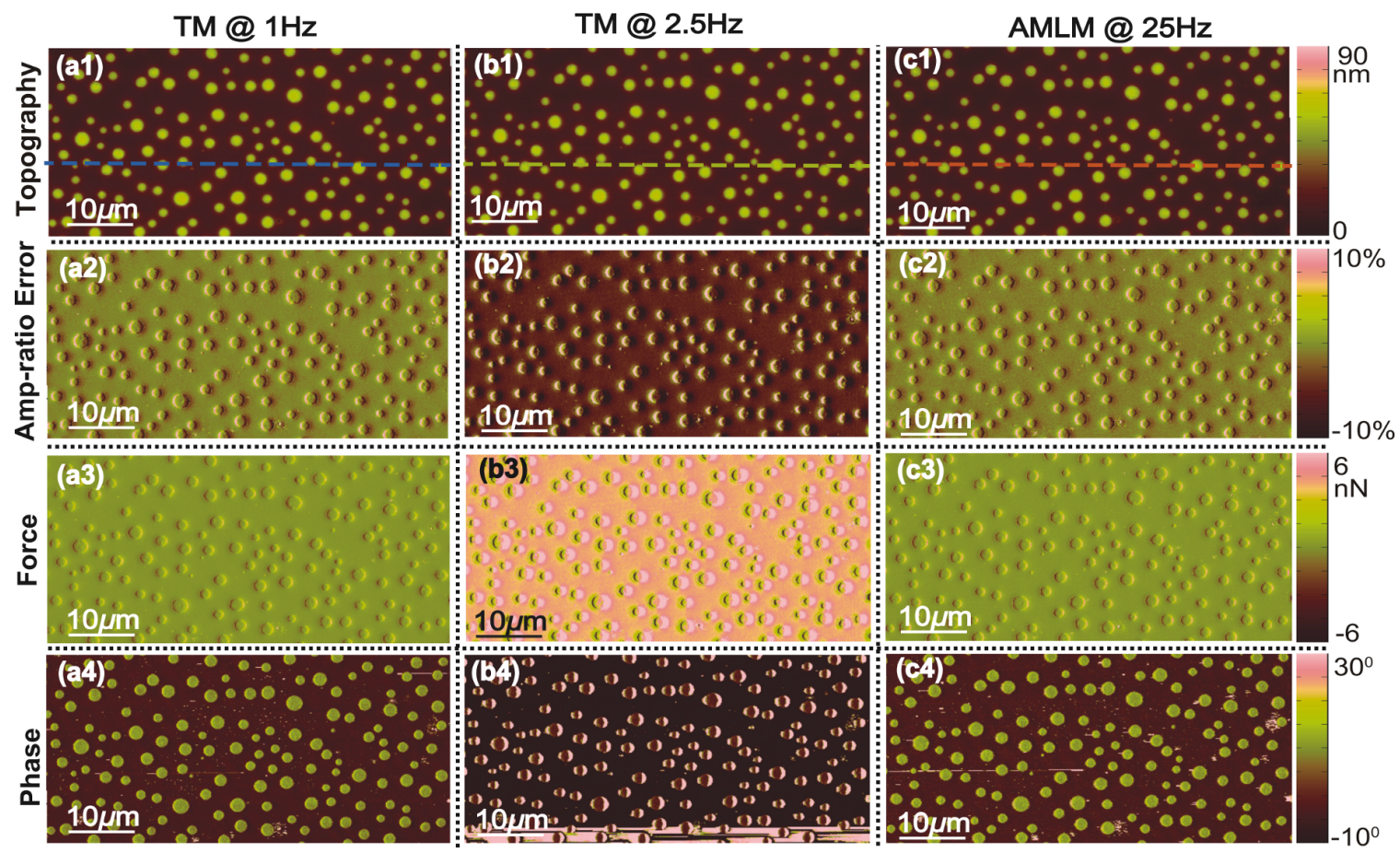

Figure 1: Images of the PS-LDPE sample topography (scan area: $50 \mu \mathrm{m} \times 25 \mu \mathrm{m}$, scan direction: $50 \mu \mathrm{m}$ ). the corresponding tapping-amplitude-ratio error, the averaged tip-sample interaction force, and the phase contrast obtained using TM imaging at (a1-a4) $1 \mathrm{~Hz}$ and (b1-b4) $2.5 \mathrm{~Hz}$, and AMLM imaging at (c1-c4) $25 \mathrm{~Hz}$, respectively.

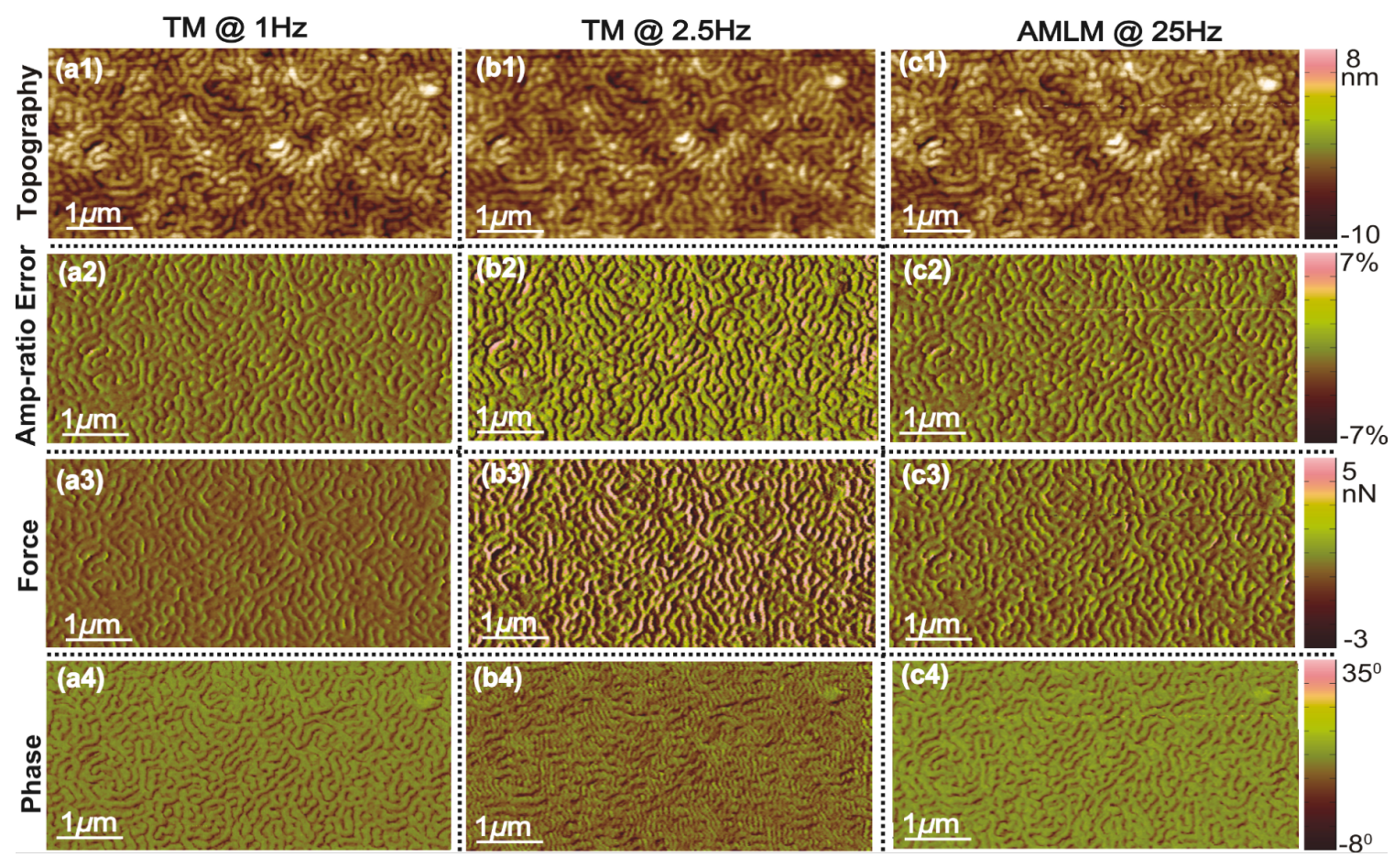

Figure 2: Images of the SBS sample topography (scan area: $5 \mu \mathrm{m} \times 2.5 \mu \mathrm{m}$, scan direction: $5 \mu \mathrm{m}$ ), the corresponding tapping-amplitude-ratio error, the averaged tip-sample interaction force, and the phase contrast obtained using TM imaging at (a1-a4) $1 \mathrm{~Hz}$ and (b1-b4) $2.5 \mathrm{~Hz}$, and AMLM imaging at (c1-c4) $25 \mathrm{~Hz}$, respectively. 


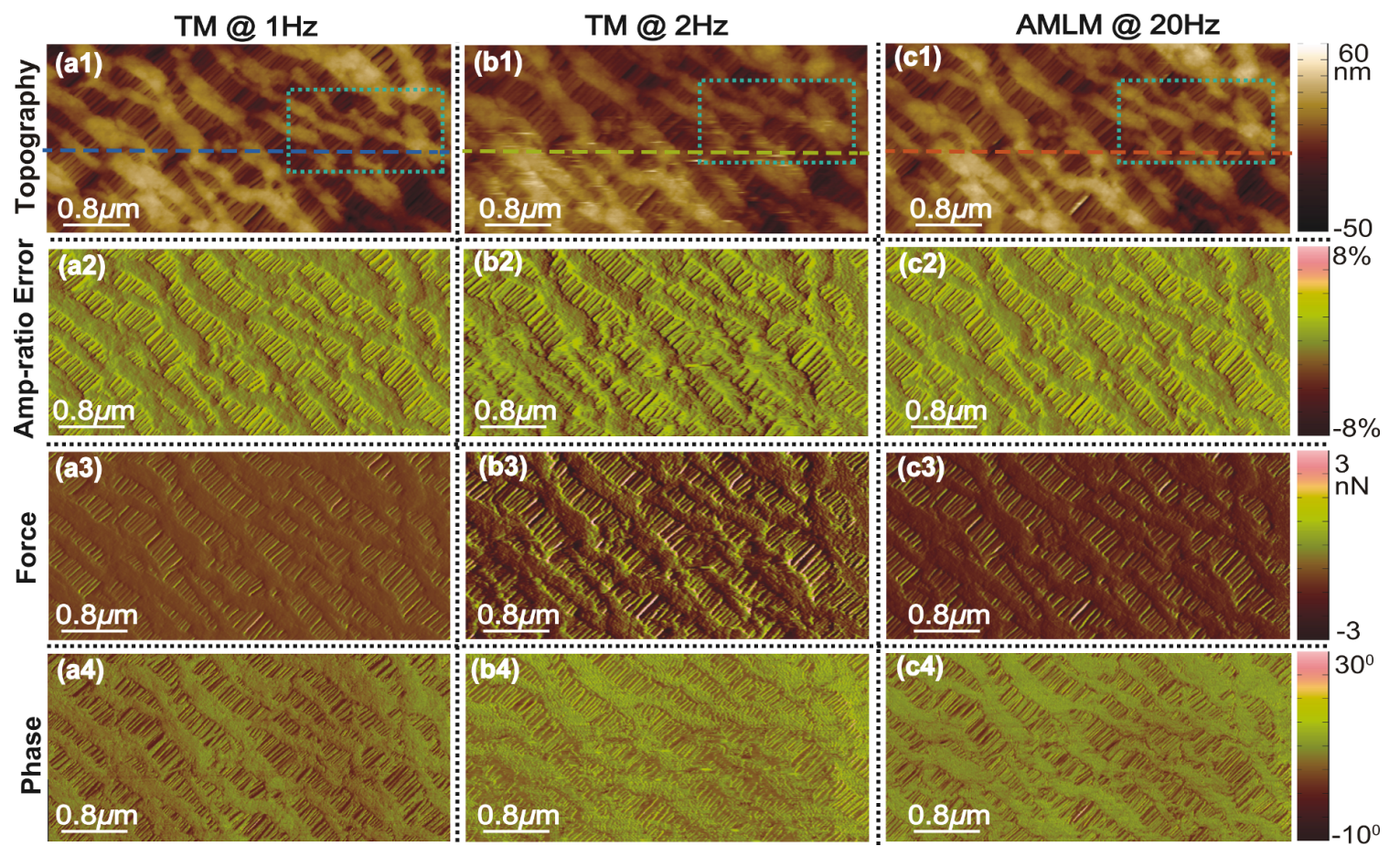

Figure 3: Images of the Celgard sample topography (scan area: $4 \mu \mathrm{m} \times 2 \mu \mathrm{m}$, scan direction: $4 \mu \mathrm{m}$ ), the corresponding tapping-amplitude-ratio error, the averaged tip-sample interaction force, and the phase contrast obtained using TM imaging at (a1-a4) $1 \mathrm{~Hz}$ and (b1 b4) $2 \mathrm{~Hz}$, and AMLM imaging at (c1-c4) $20 \mathrm{~Hz}$, respectively.

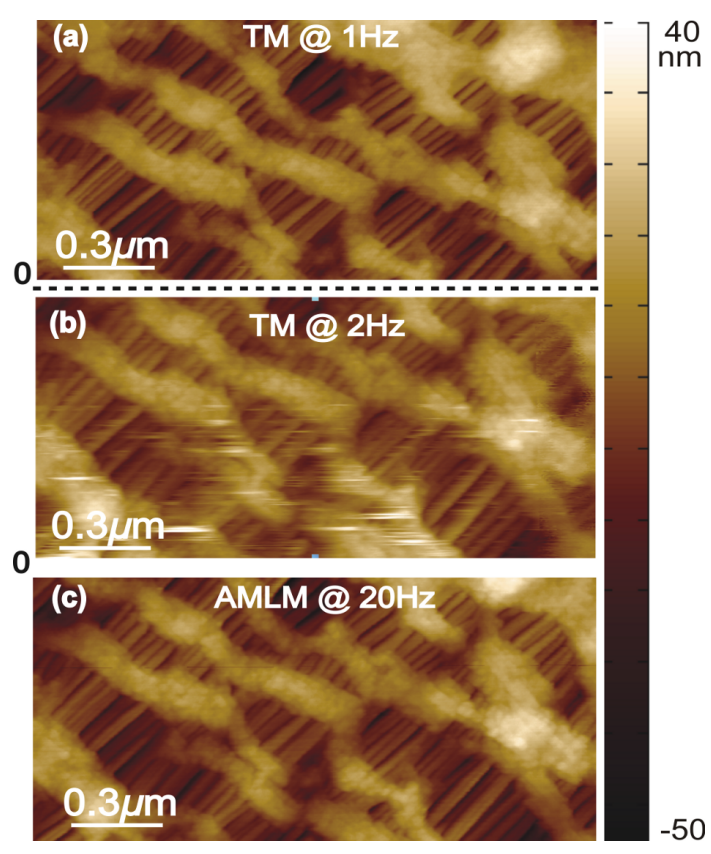

Figure 4: Zoomed-in view of the Celgard sample topography as marked out in Figure 3 obtained using TM imaging at (a) $1 \mathrm{~Hz}$ and (b) $2 \mathrm{~Hz}$, and AMLM imaging at (c) $20 \mathrm{~Hz}$.

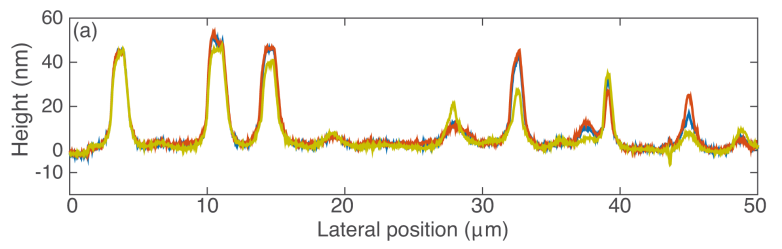

-TM @ $1 \mathrm{~Hz}$ —AMLM @ 25Hz-TM @ 2.5 Hz

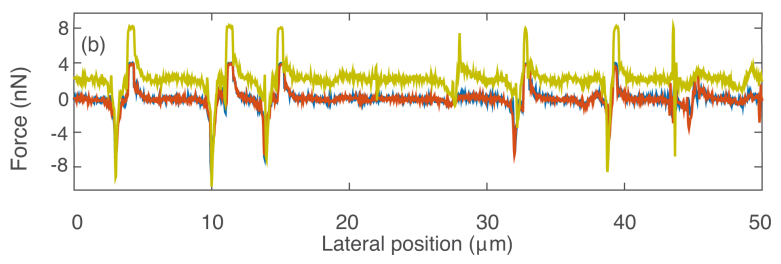

Figure 5: Comparison of (a) the sample topography and

(b) the averaged tip-sample interaction force at the cross-section marked out in Figure 1.

topography error of the PS-LDPE image obtained using AMLM imaging at $25 \mathrm{~Hz}$ was $9 \%$, which is $16 \%$ lower than that of the $2.5 \mathrm{~Hz}$ TM image. The averaged tip-sample interaction force and the tapping-amplitude-ratio error of AMLM imaging at $25 \mathrm{~Hz}$ were, respectively, $43 \%$ and $31 \%$ lower than those of the TM imaging at $2.5 \mathrm{~Hz}$. Such substantial improvements can also be clearly seen from the cross-section plot in Figure 5. At the cross-section marked in Figure 1, the topogra- 


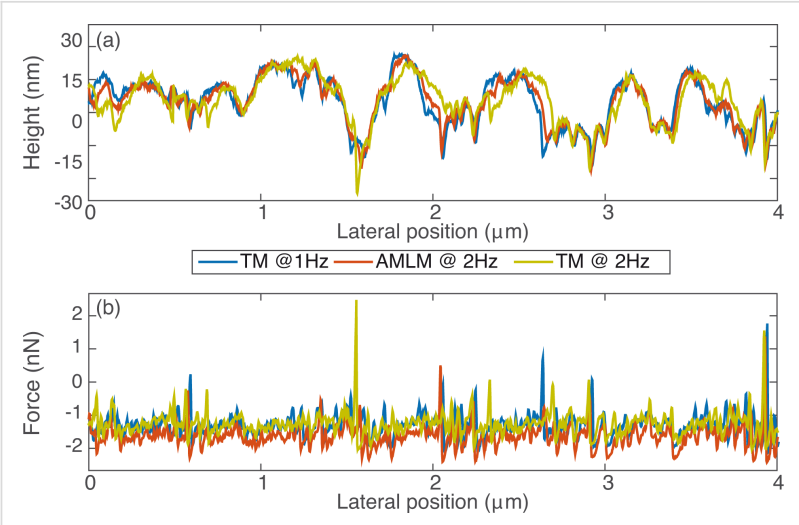

Figure 6: Comparison of (a) the sample topography and (b) the averaged tip-sample interaction force at the cross-section marked out in Figure 3.

phy imaging error of the $2.5 \mathrm{~Hz}$ TM image, with respect to the $1 \mathrm{~Hz}$ TM image, was $18 \%$ higher than that of the $25 \mathrm{~Hz}$ AMLM image of $8 \%$. As a result, the amplitude of the averaged tip-sample interaction force of the $2.5 \mathrm{~Hz} \mathrm{TM}$ imaging at that scan-line was $28 \%$ higher than that of the $25 \mathrm{~Hz}$ AMLM imaging. Note that as the imaging quality needs to be traded-off with the force applied in TM imaging (or in all AFM imaging techniques in general), to achieve the best possible TM images of the PS-LDPE sample at the scan rate of $2.5 \mathrm{~Hz}$ in experiments (as shown in Figure 1), the set-point of the amplitude ratio was experimentally tuned and set around $20 \%$, resulting in large probe-sample interaction force. Furthermore, both the phase image obtained using the TM imaging at $1 \mathrm{~Hz}$ and that obtained by AMLM imaging at $25 \mathrm{~Hz}$ clearly revealed the material property difference between PS and LDPE. In Figure 1, the round dots are made of LDPE, and the rest is PS, with the elasticity of PS and LDPE being $2 \mathrm{GPa}$ and $100 \mathrm{MPa}$, respectively. However, this information was not discernible in the $2.5 \mathrm{~Hz}$ TM phase image (see Figure 1(b4)). Therefore, AMLM imaging yielded high-quality dynamic-mode imaging, and clearly identified the material property difference of heterogeneous polymer samples at high speed and large imaging size.

The performance, usability and robustness of the AMLM technique can be further evaluated through the images of the SBS sample and the Celgard sample with much smaller features than the PS-LDPE sample, at small imaging sizes of $5 \mu \mathrm{m}$ and $4 \mu \mathrm{m}$, respectively. In Figure 2, the "squiggle" feature of the SBS sample was clearly tracked by AMLM imaging at $20 \mathrm{~Hz}$ and TM imaging at $1 \mathrm{~Hz}$, but was blurry when using TM imaging at $2.5 \mathrm{~Hz}$. The topography error of the AMLM imaging was $8 \%$ with respect to the $1 \mathrm{~Hz}$ TM imaging. Moreover, the averaged tip-sample interaction force and the tapping-amplitude-ratio error of the $25 \mathrm{~Hz}$ AMLM imaging were $14 \%$ and 17\%, respectively, different from those of the $1 \mathrm{~Hz} \mathrm{TM}$ imaging, or equiva- lently, $42 \%$ and $26 \%$, respectively, lower than those of the $2.5 \mathrm{~Hz}$ TM imaging. Due to its porous feature at nanometer scale and extremely low tensile strength, Celgard poses a rather daunting challenge for high-speed topography imaging. The experiment results demonstrated that high-quality topography images were achieved at a scan rate of $20 \mathrm{~Hz}$ using the AMLM technique. For example, the zoomed-in view of the topography images in Figure 4 showed that the porous feature (at a size around $10 \mathrm{~nm}$ ) of the Celgard sample was clearly captured in the $20 \mathrm{~Hz}$ AMLM image and the $1 \mathrm{~Hz}$ TM image, but was seriously distorted in the $2 \mathrm{~Hz} \mathrm{TM}$ image. The topography error of the AMLM imaging was $11 \%$. Also AMLM imaging was able to maintain the tip-sample interaction force and the tappingamplitude-ratio error $27 \%$ and $11 \%$ lower than those of the $2 \mathrm{~Hz} \mathrm{TM}$ imaging, respectively (both with respect to the corresponding results of the $1 \mathrm{~Hz}$ TM imaging). When comparing the sample topography tracking error and the amplitude of the averaged tip-sample interaction force at the cross-section marked out in Figure 3 the $2 \mathrm{~Hz} \mathrm{TM}$ imaging results were $41 \%$ and $27 \%$, respectively, which is $32 \%$ and $16 \%$ higher than those of the $20 \mathrm{~Hz}$ AMLM imaging results, respectively (Figure 6). Therefore, AMLM imaging is also capable of high-speed imaging on polymer samples with much smaller feature size at small scan sizes.

Moreover, to quantify the overall performance of the AMLM technique over TM imaging for all the three tested benchmark polymer samples, the tapping-amplitude-ratio error and the mean tip-sample interaction force distributions (the RMS values and the standard deviation) over the entire images are shown in Figure 7. It is clear that by using the AMLM imaging,
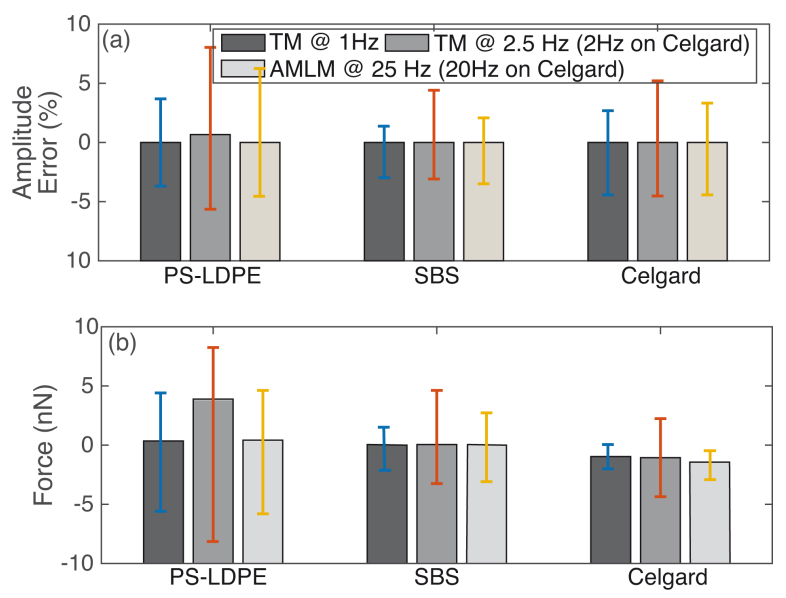

Figure 7: Comparison of (a) the tapping-amplitude-(ratio) error and (b) the averaged tip-sample interaction force over the entire image for $1 \mathrm{~Hz}$ TM imaging, $2.5 \mathrm{~Hz}(2 \mathrm{~Hz}) \mathrm{TM}$ imaging, and $25 \mathrm{~Hz}(20 \mathrm{~Hz})$ AMLM imaging of the three tested samples, where the vertical bar denotes the corresponding standard deviation over the entire image. 
the tip-sample interaction force was much better controlled than thatwhen using the TM imaging during the imaging of all three tested samples: When using the AMLM imaging, the fluctuation of the tapping amplitude ratio (measured by its RMS value) was only increased slightly by $13 \%, 17 \%$, and $8 \%$ for the PS-LDPE sample, the SBS sample and the Celgard sample, respectively, compared to that of $1 \mathrm{~Hz}$ TM imaging, and $31 \%$, $26 \%$ and $11 \%$ smaller than that of the $2.5 \mathrm{~Hz} \mathrm{TM}$ imaging, respectively. The mean tip-sample interaction force of AMLM imaging at $25 \mathrm{~Hz}$ (and $20 \mathrm{~Hz}$ ) was slightly increased by $6 \%$, $14 \%$, and $19 \%$ from that of $1 \mathrm{~Hz}$ TM imaging on the PS-LDPE sample, the SBS sample and the Celgard sample, respectively, and $43 \%, 42 \%$ and $27 \%$ lower than that of TM imaging at $2.5 \mathrm{~Hz}$ (and $2 \mathrm{~Hz}$ ), respectively. As a further comparison, the 10 $\mathrm{Hz}$ TM topography images of the SBS and the Celgard samples are also shown in Figure 8. It is clear that the $10 \mathrm{~Hz} \mathrm{TM}$ imaging results were not acceptable. Therefore, the imaging results on the three benchmark samples demonstrated the capa-

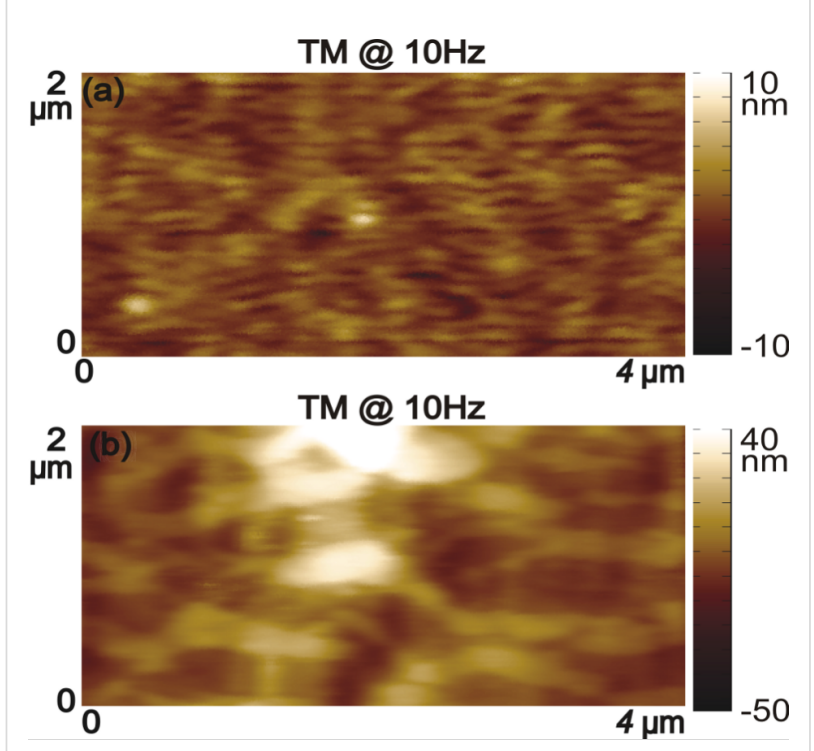

Figure 8: Topography images obtained using the TM imaging at the scan rate of $10 \mathrm{~Hz}$ of (a) the SBS sample and (b) the Celgard sample. bility of the AMLM technique in high-quality high-speed imaging on a wide variety of samples with largely different feature sizes and mechanical properties.

\section{Conclusion}

In this work, the performance of the adaptive multiloop-mode (AMLM) imaging technique has been tested and assessed via high-speed imaging of three benchmark polymer samples, namely PS-LDPE, SBS and Celgard. The AMLM imaging results at scan rates of $25 \mathrm{~Hz}$ and $20 \mathrm{~Hz}$ were compared to those obtained by using TM imaging at scan rates of $2.5 \mathrm{~Hz}$ and $2 \mathrm{~Hz}$, respectively. The imaging results demonstrated the efficacy of the AMLM imaging approach and its superiority over commercial TM imaging in both small- and large-size imaging. AMLM imaging maintained the imaging quality at scan rates of $25 \mathrm{~Hz}$ and $20 \mathrm{~Hz}$ as that of the TM imaging at $1 \mathrm{~Hz}$, and reduced the tapping-amplitude-ratio error and the mean tip-sample interaction force by over $15 \%$ and $27 \%$, respectively, from those of $\mathrm{TM}$ imaging at 10 -fold slower scan rates $(2.5 \mathrm{~Hz}$ and $2 \mathrm{~Hz})$. Therefore, the experimental results clearly demonstrated the AMLM as a highly efficient practical technique for high-speed dynamic-mode imaging of a wide variety of heterogeneous samples.

\section{Experimental}

\section{Adaptive multiloop-mode imaging}

The AMLM aims to achieve high-speed dynamic-mode imaging by precisely tracking the sample topography, while minimizing the mean tip-sample interaction force per vibration period, $\left\langle F_{\mathrm{t}-\mathrm{s}}\right\rangle$.

The key to the optimization of the mean tip-sample interaction force is to accurately track the sample topography by the AFM $z$-axis piezo. AMLM imaging introduces a feedback control of inner-outer loop structure to regulate the mean cantilever deflection per vibration period (called the TM-deflection). Thus the averaged (vertical) position of the cantilever in each tapping period is kept closely around the desired value (see Figure 9) [1]. The outer loop adjusts the TM-deflection set-point in real

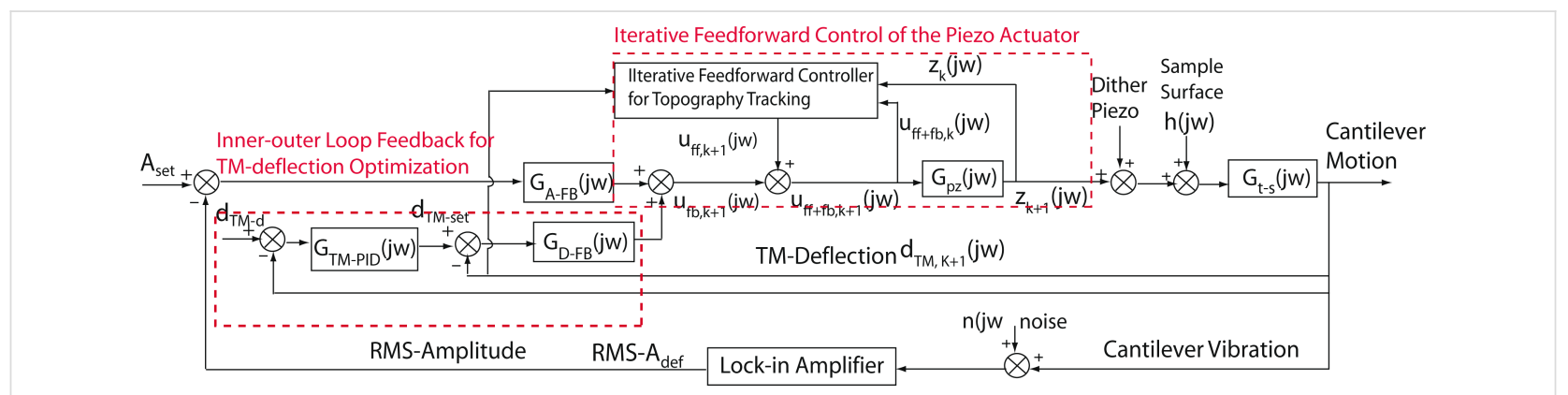

Figure 9: Schematic block diagram of the proposed AMLM imaging. This figure is an adapted version from [1]. 
time, while the inner loop tracks the adjusted TM-deflection set point. The following extended gradient-based approach is employed to adjust the TM-deflection set-point, $d_{T M-s e t}(\cdot)$,

$$
\begin{aligned}
& d_{\mathrm{TM}-\mathrm{set}}(j+1)=k_{\mathrm{I}} d_{\mathrm{TM}-\mathrm{set}}(j)+k_{\mathrm{P}} e_{\mathrm{TM}}(j) \\
& +k_{\mathrm{D}}\left[e_{\mathrm{TM}}(j-1)-e_{\mathrm{TM}}(j)\right] \\
& \text { with } e_{\mathrm{TM}}(j)=d_{\mathrm{TM}-\mathrm{d}}-d_{\mathrm{TM}}(j) \text {, and } j=2 \ldots N-1 \text {, }
\end{aligned}
$$

where $N$ is the total number of sampling periods per image, $d_{\mathrm{TM}}(j)$ is the TM-deflection of the current sampling point, and $k_{\mathrm{P}}, k_{\mathrm{I}}$, and $k_{\mathrm{D}}$ are the proportional (P), integral (I), and derivative (D) parameters, respectively. The desired TM-deflection, $d_{\mathrm{TM}-\mathrm{d}}$, is determined by the ratio of the chosen tapping-amplitude set-point to the free amplitude, $A_{\text {set }} / A_{\text {free }}$, based on a priori measured relation between $d_{\mathrm{TM}-\mathrm{d}}$ and $\left(A_{\mathrm{def}} / A_{\text {free }}\right)$ [1].

An online data-driven iterative feedforward controller is integrated to the RMS- $z$-feedback loop to enhance the sample topography tracking (Figure 9). Particularly, the feedforward control input is obtained by implementing the following highorder modeling-free difference-inversion-based iterative-control (HOMDIIC) algorithm [1,17] online,

$U_{\mathrm{ff}, 0}(j \omega)=0$,

$U_{\mathrm{ff}, 1}(j \omega)=\frac{U_{\mathrm{ff}+\mathrm{fb}, 0}(j \omega)}{Z_{0}(j \omega)} H_{\mathrm{ffd}, 1}(j \omega)$,

$U_{\mathrm{ff}, k+1}(j \omega)=U_{\mathrm{ff}, \mathrm{k}}+\frac{U_{\mathrm{ff}+\mathrm{fb}, k}(j \omega)-U_{\mathrm{ff}+\mathrm{fb}, k-1}(j \omega)}{Z_{k}(j \omega)-Z_{k-1}(j \omega)} e_{k}(j \omega)$,

for $k \geq 1$, and $e_{k}(j \omega)=H_{\mathrm{ffd}, k+1}(j \omega)-Z_{k}(j \omega)$

where " $j \omega$ " denotes the Fourier transform of the corresponding signal, and $U_{\mathrm{ff}+\mathrm{fb}, k}(\cdot)$ and $Z_{k}(\cdot)$ are the total control input (feedback+feedforward) applied to the $z$-piezo actuator (i.e., $U_{\mathrm{ff}+\mathrm{fb}, k}(j \omega)=U_{\mathrm{ff}, k}(j \omega)+U_{\mathrm{fb}, k}(j \omega)$, see Figure 9), and the $z$-piezo displacement measured on the $k$-th scan line, respectively, and $H_{\mathrm{ffd}, k+1}(\cdot)$ denotes the desired trajectory that the $z$-piezo needs to track at the $(k+1)$-th scanline. Note that the ratio in the above control law, $\left(U_{\mathrm{ff}+\mathrm{fb}, k}(j \omega)-U_{\mathrm{ff}+\mathrm{fb}, k-1}(j \omega)\right) /\left(Z_{k}(j \omega)-\right.$ $\left.Z_{k-1}(j \omega)\right)$, essentially equals the inverse of the frequency response of the $z$-piezo actuator, and is updated line-by-line iteratively throughout the whole imaging process.

Furthermore, the design of $H_{\mathrm{ffd}, k}(\cdot)$ in Equation 2 takes into account both the sample topography of the current scan line and the TM-deflection tracking error, $d_{\mathrm{TM}, k}(j)-d_{\mathrm{TM}-\mathrm{d}}[1]$ as follows

$$
h_{\mathrm{ffd}, k+1}(j)=h_{k}(j)+\alpha\left[d_{\mathrm{TM}, k}(j)-d_{\mathrm{TM}-\mathrm{d}}\right], j=1 \ldots N_{1}
$$

Here, $N_{1}$ and $\alpha$ are the total sampling points per scan-line and the correction factor, respectively. Details of implementation the iterative scheme in AMLM can be found in [1].

As the TM-deflection responds much faster than the RMS tapping-amplitude to the sample topography variation, so the inner-outer loop feedback control of the TM-deflection is faster than that of the RMS tapping amplitude. Moreover, the TM-deflection feedback loop facilitates the regulation of the tapping amplitude, and further minimizes the averaged probe-sample interaction force (by regulating the TM-deflection closely around the desired value). The feedforward controller further reduces the fluctuation of the tapping amplitude upon sudden sample topography variation.

To ensure the accuracy of sample topography quantification, AMLM imaging takes both the AFM $z$-piezo displacement and the TM-deflection into account [1] in quantification of $h_{k}(j)$. In particular, the sample surface height at any given point can be quantified with respect to a fixed reference point with $h_{0}=0$ and the corresponding $d_{\mathrm{TM}, 0}=d_{\mathrm{TM}-\mathrm{d}}[1]$,

$$
h_{k}(j)=z_{k}(j)+\varepsilon\left[d_{\mathrm{TM}, k}(j)-d_{\mathrm{TM}-\mathrm{d}}\right]
$$

where $\varepsilon$ is the contact constant that depends on the tip-sample interaction regime: $\varepsilon=-1$ when the tip-sample interaction is dominated by the long-range attractive force. $\varepsilon=1$ when a repulsive tip-sample interaction force appears. For $-1 \ll \varepsilon<0$ the interaction force vanishes (i.e., the tapping amplitude is close to the free vibration amplitude).

Finally, the mean tip-sample interaction force (per tapping period $T),\left\langle F_{\mathrm{t}-\mathrm{s}}\right\rangle$, can be quantified as $[1,10,11]$,

$$
\left\langle F_{\mathrm{t}-\mathrm{s}}\right\rangle=(1 / T) \oint F_{\mathrm{t}-\mathrm{s}}(t) \mathrm{d} t=k_{\mathrm{c}} d_{\mathrm{TM}},
$$

where $F_{\mathrm{t}-\mathrm{s}}(t)=m \ddot{d}_{\mathrm{tot}}(t)+\frac{m \omega_{0}}{Q} \dot{d}_{\text {tot }}(t)+k_{\mathrm{c}} d_{\mathrm{tot}}(t)$,

and $d_{\mathrm{tot}}(t)=d_{\mathrm{TM}}(t)+\left[A_{\mathrm{def}} \cos \left(\omega_{0} t+\phi\right)-A_{\text {free }} \cos \left(\omega_{0} t\right)\right]$.

where $k_{\mathrm{c}}$ and $m$ are the spring constant and the mass of the cantilever, respectively, $Q$ is the quality factor of the cantilever, $d_{\text {tot }}(t), A_{\text {def }}$ and $A_{\text {free }}$ are the total deflection, the tapping amplitude, and the free-vibration amplitude of the cantilever, respectively, and $\omega_{0}$ is the tapping frequency.

\section{Implementation of AMLM imaging}

To test and evaluate the AMLM imaging technique, three benchmark samples (a PS-LDPE sample, an SBS sample, and a Celgard sample) were imaged using the AMLM approach on an 
AFM system (Dimension Icon, Bruker Nano Inc.) along with a tapping-mode cantilever (MPP-21120-10, Bruker Inc., nominal spring constant: $3 \mathrm{~N} / \mathrm{m}$, resonant frequency: $75 \mathrm{kHz}$ ). Both the conventional TM imaging and AMLM imaging were designed and implemented in a Matlab xPC-target (R2010a) system, and applied to the AFM imaging through a computer-based data acquisition system (NI-6259, National Instrument, sampling frequency: $20 \mathrm{kHz}$ ). The AFM system was modified to allow for direct acquisition of all the required sensor signals and direct application of the driven signals to the AFM piezo actuators. Before imaging, the HODMIIC technique (Equation 2) was employed to achieve precise tracking in the lateral $x-y$ axes scanning [17] (the RMS tracking error was maintained below 1\%). Then the converged inputs for $x$ - and $y$-piezo actuators were applied during the imaging. Tapping at $30 \%$ of the free vibration of $120 \mathrm{~nm}$ was chosen so that the tapping motion of the cantilever was maintained and the corresponding mean TM-deflection was closely around zero, thereby, minimizing the corresponding mean vertical probe-sample interaction force. To make a fair comparison between AMLM and TM, the amplitude set-point in all TM imaging was not fixed and tuned in order to get the best possible images.

\section{Acknowledgements}

The financial support of NSF CAREER award CMMI-1066055 and NSF grant CMMI-1200557 is gratefully acknowledged. We also thank Dr. Chanmin Su and Dr. Shuiqing Hu from Bruker Nano, Inc. for providing the samples.

\section{References}

1. Ren, J.; Zou, Q.; Li, B.; Lin, Z. Phys. Rev. E 2014, 90, 012405. doi:10.1103/PhysRevE.90.012405

2. Khurshudov, A. G.; Kato, K.; Koide, H. Wear 1997, 203-204, 22-27. doi:10.1016/S0043-1648(96)07447-9

3. Bar, G.; Thomann, Y.; Brandsch, R.; Cantow, H.-J.; Whangbo, M.-J. Langmuir 1997, 13, 3807-3812. doi:10.1021/la970091m

4. Butt, H.-J.; Cappella, B.; Kappl, M. Surf. Sci. Rep. 2005, 59, 1-152. doi:10.1016/j.surfrep.2005.08.003

5. Sulchek, T.; Hsieh, R.; Adams, J. D.; Yaralioglu, G. G.; Minne, S. C.; Quate, C. F.; Cleveland, J. P.; Atalar, A.; Adderton, D. M. Appl. Phys. Lett. 2000, 76, 1473-1475. doi:10.1063/1.126071

6. Sulchek, T.; Yaralioglu, G. G.; Quate, C. F.; Minne, S. C. Rev. Sci. Instrum. 2002, 73, 2928-2936. doi:10.1063/1.1488679

7. Sahoo, R. D.; Sebastian, A.; Salapaka, M. V. Appl. Phys. Lett. 2004, 83, 5521-5523. doi:10.1063/1.1633963

8. Huang, P.; Andersson, S. B. Appl. Phys. Lett. 2013, 102, 213118. doi:10.1063/1.4808211

9. Balantekin, M.; Satır, S.; Torello, D.; Değertekin, F. L. Rev. Sci. Instrum. 2014, 85, 123705. doi:10.1063/1.4903469

10. San Paulo, A.; García, R. Phys. Rev. B 2001, 64, 193411. doi:10.1103/PhysRevB.64.193411

11. Su, C.; Huang, L.; Kjoller, K. Ultramicroscopy 2004, 100, 233-239. doi:10.1016/j.ultramic.2003.11.007
12. Rogers, B.; Sulchek, T.; Murray, K.; York, D.; Jones, M.; Manning, L.; Malekos, S.; Beneschott, B.; Adams, J. D.; Cavazos, H.; Minne, S. C. Rev. Sci. Instrum. 2003, 74, 4683-4686. doi:10.1063/1.1619548

13. Picco, L. M.; Bozec, L.; Ulcinas, A.; Engledew, D. J.; Antognozzi, M.; Horton, M. A.; Miles, M. J. Nanotechnology 2007, 18, 044030. doi:10.1088/0957-4484/18/4/044030

14. Bruker-Corp.. Dimension FastScan - Learn more - Bruker. http://www.bruker.com/products/surface-analysis/atomic-force-microsc opy/dimension-fastscan/learn-more.html (accessed June 30, 2017).

15. Kodera, N.; Sakashita, M.; Ando, T. Rev. Sci. Instrum. 2006, 77, 083704. doi:10.1063/1.2336113

16. Sahoo, D. R.; Agarwal, P.; Salapaka, M. V. Transient force atomic force microscopy: A new nano-interrogation method. American Control Conference 2007; 2007; pp 2135-2140.

17. Wang, Z.; Tan, J.; Zou, Q.; Jiang, W. Rev. Sci. Instrum. 2013, 84 , 113704. doi: $10.1063 / 1.4832046$

\section{License and Terms}

This is an Open Access article under the terms of the Creative Commons Attribution License

(http://creativecommons.org/licenses/by/4.0), which permits unrestricted use, distribution, and reproduction in any medium, provided the original work is properly cited.

The license is subject to the Beilstein Journal of

Nanotechnology terms and conditions:

(http://www.beilstein-journals.org/bjnano)

The definitive version of this article is the electronic one which can be found at: doi:10.3762/bjnano.8.158 